\title{
ON THE SUBALGEBRAS OF CERTAIN FINITELY PRESENTED ALGEBRAS
}

\author{
BY GILBERT BAUMSLAG ${ }^{1}$ \\ Communicated by Barbara L. Osofsky, October 3, $1975^{2}$
}

1. Every finitely generated metabelian group can be embedded in a finitely presented metabelian group [2]. The object of this note is to announce some theorems of a similar nature for lie and associative algebras. Since associative algebras are structurally richer than groups, it is not surprising that the associative algebra versions of these theorems (see \$2) encompass somewhat more than the corresponding known theorems for groups (see [5], [2], and [8]). The same cannot, however, be said at this time for lie algebras (see §3).

2. All of our embedding theorems for associative algebras stem from a simple criterion, Theorem A (below), for an associative algebra to be finitely presented. In order to explain, we need to introduce some notation and definitions. Let then $T$ be an associative algebra over (as always) a commutative field. If $n$ is any positive integer, then $T^{n}$ is the subalgebra of $T$ generated by all $n$-fold products of elements of $T$; notice that $T^{n}$ is an ideal of $T . T$ is termed nilpotent if $T^{n}=0$ for some $n$. Now let $M$ be a $(T, T)$-bimodule. We term $M$ ample if for each $m \in M, t \in T$, there exist $t^{\prime}, t^{\prime \prime} \in T$ such that $m t=$ $t^{\prime} m, t m=m t^{\prime \prime}$. The following theorem then holds.

TheOREM A. Let $0 \rightarrow N \rightarrow A \rightarrow T \rightarrow 0$ be a short exact sequence of associative algebras, where $N$ is nilpotent and all of the quotients of $T$ are finitely presented. If $N^{i} / N^{i+1}$, viewed as a $(T, T)$-bimodule, is finitely generated and ample for every $i$, then $A$ is finitely presented.

On appealing to a theorem of J. Lewin [7], Theorem A can be used to deduce

THEOREM B. Let $A$ be a finitely generated associative algebra containing an ideal $N$ with $N^{2}=0$. If every quotient of $A / N$ is finitely presented then $A$ can be embedded in a finitely presented algebra $A^{*}$ which closely resembles $A$; specifically $A^{*}$ contains an ideal $N^{*}$ such that (i) $N^{* 2}=0$ and (ii) $A^{*} / N^{*}$ $=A / N \otimes A / N$.

AMS (MOS) subject classifications (1970). Primary 16A06, 16A46, 16A56; Secondary 17B35, 17B65.

${ }^{1}$ Support from the National Science Foundation is gratefully acknowledged.

2 Originally received August 30, 1975. 
Theorem B has a number of interesting consequences.

Corollary B1. Let $A$ be a finitely generated associative algebra which is the middle of a short exact sequence $0 \rightarrow N \rightarrow A \rightarrow T \rightarrow 0$ in which $N^{2}=0$ and $T$ is lie-nilpotent. Then $A$ can be embedded in a finitely presented algebra $A^{*}$ which is also the middle of a short exact sequence of exactly the same kind.

It may be as well to remind the reader of the definition of lie-nilpotent. To this end recall that if $A$ is any associative algebra, then $A$ becomes a lie algebra, denoted $\left(A,{ }^{\circ}\right)$, if we replace the multiplication in $A$ by the multiplication

$$
a \circ b=a b-b a \quad(a, b \in A) .
$$

Then $A$ is lie-nilpotent if the lie algebra $\left(A,{ }^{\circ}\right)$ is nilpotent (see e.g. N. Jacobson $[4$, p. 25]). Among the lie-nilpotent algebras are the commutative algebras. So a special case of Corollary B1 is

Corollary B2. Suppose the finitely generated associative algebra $A$ contains an ideal $N$ such that $N^{2}=0$ and $A / N$ is commutative. Then $A$ can be embedded in a finitely presented algebra $A^{*}$ containing an ideal $N^{*}$ such that $N^{* 2}=0$ and $A^{*} / N^{*}$ is commutative.

Corollary B2 may be regarded as an analogue of the embedding theorem for finitely generated metabelian groups cited at the outset.

We turn now to a slightly different run-off from Theorem A, viz.

THEOREM C. Let $A$ be a finitely generated algebra of lower triangular matrices with coefficients in a commutative algebra $B$. Then $A$ can be embedded in a finitely presented algebra of lower triangular matrices with coefficients in $B$.

The analogue of Theorem $\mathrm{C}$ has recently been obtained for groups by M. Thomson [8].

Our last embedding theorem for associative algebras is

THEOREM D. Let $F$ be a finitely generated free associative algebra and let $R$ be an ideal of $F$. If $F / R$ is finitely presented, then $F / R^{n}$ can be embedded in a finitely presented algebra $A$ containing an ideal $S$ such that (i) $S^{n}=0$ and (ii) $A / S=F / R \otimes F / R$.

Unlike Theorem $\mathrm{C}$ no analogue of Theorem $\mathrm{D}$ has as yet been proved for groups.

3. We turn now to lie algebras over a commutative field of characteristic different from 2. Here our results are much more restricted than those for associative algebras described above. In order to explain, let $L$ be a lie algebra with multiplication denoted as usual by $[] .$,$L is said to be metabelian if$ 
the second term of its derived series (see e.g. N. Jacobson [4, p. 23]) is trivial, i.e. if $L^{\prime \prime}=0$. Then we have the following lie algebra analogue of the main theorem in [2]:

THEOREM E. A finitely generated metabelian lie algebra over a field of characteristic different from 2 can be embedded in a finitely presented metabelian lie algebra.

Theorem E may also be viewed as an analogue of Corollary B2.

Theorem $\mathrm{C}$, too, has a lie algebra analogue:

THEOREM F. Let $L$ be a finitely generated lie algebra of lower triangular matrices with coefficients in a commutative algebra $B$. Then $L$ can be embedded in a finitely presented lie algebra of lower triangular matrices with coefficients in $B$.

4. In conclusion I would like to prove a special case of Theorems B, C and $D$. The idea involved in this proof is central in the proofs of all of the theorems described in $\$ 2$. There is a vaguely similar, but more complicated, special case of the lie algebra theorems in $\$ 3$. We shall, however, not record that proof here, remarking only that Theorem $\mathrm{E}$ depends on the main theorem of R. K. Amayo and Ian Stewart [1].

Let now $F$ be the free associative algebra on $x$ and $y$ (over some commutative field). Furthermore let $R$ be the ideal of $F$ generated by $x$. Then $F / R$ is a polynomial algebra in a single variable $y+R$ and hence it is not finite dimensional. So by a theorem of J. Lewin [6] $F / R^{2}=A$ (say) is not finitely presented! Our objective is to embed $A$ in a finitely presented algebra $A^{*}$ of the same kind. To this end, put $a=x+R^{2}, b=y+R^{2}$. Then it is not hard to see that $A$ can be presented on $a$ and $b$ subject to the defining relations $a b^{i} a=0(i=0,1, \ldots)$ :

$$
A=\left\langle a, b ; a b^{i} a=0(i=0,1, \ldots)\right\rangle
$$

Adding another generator $c$ to this presentation and three extra relations we obtain a presentation for an algebra $A^{*}$, say:

$$
A^{*}=\left\langle a, b, c ; a b^{i} a=0(i=0,1, \ldots), c b=b c, a c=b a, c a=a b\right\rangle .
$$

It is easy to see, by using split extensions, that $A$ is a subalgebra of $A^{*}$; moreover, on appealing to Theorem $\mathrm{A}$, it turns out that $A^{*}$ is finitely presented. We shall, however, prove this assertion directly here because this proof goes straight to the heart of all of the theorems in $\$ 2$. In fact we claim that $A^{*}$ can be presented in the form

$$
A^{*}=\left\langle a, b, c ; a^{2}=0, c b=b c, a c=b a, c a=a b\right\rangle ;
$$


this is actually clear because the omitted relations $a b^{i} a=0(i=0,1, \ldots)$ follow from the ones that we have retained:

$$
a b^{i} a=\left(a b^{i}\right) a=\left(c^{i} a\right) a=c^{i} a^{2}=0 .
$$

\section{REFERENCES}

1. R. K. Amayo and Ian Stewart, Finitely generated Lie algebras, J. London Math. Soc. (2) 5 (1972), 697-703. MR 48 \#2205.

2. Gilbert Baumslag, Subgroups of finitely presented metabelian groups, J. Austral. Math. Soc., 16 (1973), 98-110. MR 48 \#11324.

3. L. A. Bokut', Unsolvability of the equality problem and subalgebras of finitely presented Lie algebras, Izv. Akad. Nauk SSSR Ser. Mat. 36 (1972), 1173-1219 = Math. USSR Izv. 6 (1972), 1153-1199. MR 48 \#8588.

4. Nathan Jacobson, Lie algebras, Interscience Tracts in Pure and Appl. Math., no. 10, Interscience, New York, 1962. MR 26 \#1345.

5. Graham Higman, Subgroups of finitely presented groups, Proc. Roy. Soc. London Ser. A 262 (1961), 455-475. MR 24 \#A152.

6. Jacques Lewin, On some infinitely presented associative algebras, J. Austral. Math. Soc. 16 (1973), 290-293. MR 49 \#5084.

7. - A matrix representation for associative algebras. I, II, Trans. Amer. Math. Soc. 188 (1974), 293-308, 309-317. MR 49 \#2848.

8. Michael Thomson, Embedding theorems for finitely generated solvable groups, Ph.D. Thesis, City University of New York, 1976.

DEPARTMENT OF MATHEMATICS, CITY COLLEGE OF NEW YORK (CUNY), NEW YORK, NEW YORK 10031 